\title{
Developmental Roles of D-bifunctional Protein- A Zebrafish Model of Peroxisome Dysfunction
}

\author{
Yong-II Kim ${ }^{1,4}$, Sushil Bhandari ${ }^{1,4}$, Joon No Lee ${ }^{1,4}$, Kyeong-Won Yoo ${ }^{1,2}$, Se-Jin Kim ${ }^{1}$, Gi-Su Oh ${ }^{1}$, \\ Hyung-Jin Kim ${ }^{1}$, Meyoung $\mathrm{Cho}^{3}$, Jong-Young Kwak ${ }^{2}$, Hong-Seob So', Raekil Park ${ }^{1, *}$, and \\ Seong-Kyu Choe ${ }^{1, *}$
}

\begin{abstract}
The peroxisome is an intracellular organelle that responds dynamically to environmental changes. Various model organisms have been used to study the roles of peroxisomal proteins in maintaining cellular homeostasis. By taking advantage of the zebrafish model whose early stage of embryogenesis is dependent on yolk components, we examined the developmental roles of the D-bifunctional protein (Dbp), an essential enzyme in the peroxisomal $\beta$ oxidation. The knockdown of $d b p$ in zebrafish phenocopied clinical manifestations of its deficiency in human, including defective craniofacial morphogenesis, growth retardation, and abnormal neuronal development. Overexpression of murine Dbp rescued the morphological phenotypes induced by $d b p$ knockdown, indicative of conserved roles of Dbp during zebrafish and mammalian development. Knockdown of $d b p$ impaired normal development of blood, blood vessels, and most strikingly, endodermderived organs including the liver and pancreas - a phenotype not reported elsewhere in connection with peroxisome dysfunction. Taken together, our results demonstrate for the first time that zebrafish might be a useful model animal to study the role of peroxisomes during vertebrate development.
\end{abstract}

\section{INTRODUCTION}

Peroxisomes perform crucial functions to maintain cellular homeostasis (Islinger et al., 2012; Van Veldhoven, 2010). Animal models of peroxisome dysfunction, at the level of its biogenesis

${ }^{1}$ Center for Metabolic Function Regulation, and Department of Microbiology, School of Medicine, Wonkwang University, Iksan 570-749, Korea, ${ }^{2}$ Immune-network Pioneer Research Center, Department of Biochemistry, College of Medicine, Dong-A University, Busan 602-714, Korea, ${ }^{3}$ Department of Internal Medicine, Gunsan Medical Center, Gunsan 573$713 \mathrm{Korea},{ }^{4}$ These authors contributed equally to this work.

*Correspondence: seongkyu642@wku.ac.kr (SKC); rkpark@wku.ac.kr (RP)

Received 17 October, 2013; revised 8 November, 2013; accepted 8 November, 2013; published online 27 January, 2014

Keywords: D-bifunctional protein, embryogenesis, model organism, peroxisome, zebrafish or separately at an individual enzyme level, were generated to elucidate the molecular mechanisms responsible for disease phenotypes in humans (Baes and Van Veldhoven, 2012). In particular, knockout mouse models provided convincing evidence showing that peroxisome is an active, multifunctional organelle communicating in response to environmental changes with other cellular compartments for maintaining normal physiological functions (Baes and Veldhoven, 2012; Faust et al., 2011; Huyghe et al., 2006). In mice, peroxisome dysfunction affects mitochondrion and Endoplasmic Reticulum (Thoms et al., 2009). However, mice develop in utero and receive nutrients from their mother through the umbilical cord during embryogenesis, making it difficult to gain adequate access to developing embryos. Additionally, such dependence makes it difficult to interpret developmental changes associated with metabolic alterations contributed solely by peroxisome dysfunction. The zebrafish is a well-established genetic and developmental model for studying human-diseases, particularly since its embryos are transparent and develop fast ex utero consuming only the yolk during the initial stages of development (Lieschke and Currie, 2007).

In this study, we examined whether zebrafish embryogenesis can be used as an effective in vivo model for studying peroxisome function, which might permit an organism-level analysis while providing mechanistic insights into development. Specifically, we analyzed the developmental roles of D-bifunctional protein (DBP, also known as 17- $\beta$-hydroxysteroid dehydrogenase 4), a peroxisomal enzyme involved in $\beta$-oxidation of fatty acids that generates acetyl-CoA and short-chain acyl CoA for intracellular energy and metabolic homeostasis (Moller et al., 2001). Mutations in humans may lead to DBP deficiency in which severe neonatal abnormalities, including growth retardation, neuropathy, craniofacial malformation, and hypotonia occur within an early period of life (Mehtälä et al., 2013; Moller et al., 2001; Suzuki et al., 1997; Van Grunsven et al., 1998; 1999). Mice deficient in $D b p$ exhibited a similar cohort of phenotypes as those found in humans that included severe growth retardation, male infertility and massive mortality within the first two postnatal weeks (Baes et al., 2000; Ferdinandusse et al., 2005; Huyghe et al., 2006; Verheijden et al., 2013). At a molecular level, DBP is the primary enzyme in the peroxisomal $\beta$-oxidation pathway that processes both saturated very long chain and branched chain fatty acids.

Here we report that $d b p$ knockdown in zebrafish embryos 
Table 1. Primer list and its sequences used in real-time PCR, cDNA cloning of $d b p$, or efficacy test for $d b p$ splicing-blocking morpholino

\begin{tabular}{|c|c|c|}
\hline Gene name & Forward primer $\left(5^{\prime}\right.$ to $\left.3^{\prime}\right)$ & Reverse primer $\left(5^{\prime}\right.$ to $\left.3^{\prime}\right)$ \\
\hline$m D b p$ & ACCGGTATGGCTTCGCCGCTGAGGTTCG & ACCGGTTCAGAGCTTGGCATAGTCTTTAAG \\
\hline$d b p$ & CGGTCTATCCTGGTCAGTCT & ТТТССССАТСАССАССТССА \\
\hline gnpatl & CGTCTTCCTCAGAGCCATTC & САCTCTGAACTCGTTCTGGTC \\
\hline agps & GTCСTCСАТАTTCAССTСАTTC & САСТСТСТТАТТАТССТСТССТ \\
\hline pex5 & GGGAGAGAGAGAAAGAGAGT & ACAGAGAGTGCGGCAGAGAAA \\
\hline pgcla & ССТССТTССТСТСАGСТСТ & СССТСТСАСАTTCCСGTTTC \\
\hline pparab & GGAGAAGCTAAGGTTGAAGG & GGGCACAGATTTGGGGAATTC \\
\hline esrra & GTTCTGGATGAGGAGATGTC & TGAGGAGAGGCAGGGTGAG \\
\hline$\beta$-actin & ACCTCATGAAGATCCTGACC & TGCTAATCCACATCTGCTGG \\
\hline$d b p-C$ & TCTCCAATCCTCCACGGTTTGTGTT & CGTTCCTCAAACTATGAGCGTTAGACAGA \\
\hline$d b p-E 1$ & САTCTCATCATGTCTGTGCC & \\
\hline$d b p-E 4$ & & СТТСТСТССАТССТССАСТ \\
\hline
\end{tabular}

leads to morphological malformations, defective yolk consumption, abnormal neuronal development, and growth retardation phenotypes similar to those observed in humans with $D B P$ mutations. A detailed analysis also revealed phenotypes not found earlier in humans or mice, including defects in the formation of blood, blood vessels, and cartilage. Strikingly, dbp knockdown nearly blocked digestive organ development. This is probably a result of reduced transcriptional activities critical for functional peroxisomes as well as mitochondrial biogenesis. These morphological phenotypes were rescued by the expression of murine $D b p$, indicating its functional conservation between zebrafish and mouse. Taken together, our results demonstrate that zebrafish is a promising animal model that may be used to uncover new roles of peroxisomes during vertebrate development. Our study is the first to demonstrate the developmental consequences of peroxisome dysfunctions by using the zebrafish model.

\section{MATERIALS AND METHODS}

Zebrafish care, transgenic animals, and microinjections The zebrafish and their embryos were handled and staged according to standard protocols (Kimmel et al., 1995). Transgenic zebrafish lines were kindly provided by Drs. C-H Kim, HC Park, Y-K Bae, Y Kee and S-Y Choi [islet1:GFP, gfap:EGFP, mbp:EGFP, gata1:RFP, MLS (mitochondria localization sequence)-EGFP], and by the Zebrafish Organogenesis and Mutant Bank (Ifabp:DSRED and elastase:GFP). Control (5'AACATACATCAGTTTAATATATGTA-3'), splicing-blocking (5'TCGGTGATGAAGAACTGACCTCCGC-3') and translationblocking (5'-CGTCGAATCTCAAAGGCACAGACAT-3') morpholinos (MOs) were purchased from Gene Tools. Approximately 8 $\mathrm{ng} \mathrm{MO}$ was microinjected at the 1-cell stage of wild type or transgenic zebrafish embryos as previously described (Choe et al., 2011). For rescue experiment, $50 \mathrm{pg}$ in vitro synthesized murine Dbp mRNA (Ambion) was co-injected with $d p b \mathrm{MO}$ and resulting phenotypes were documented.

\section{Constructs}

Murine Dbp was amplified using a standard PCR protocol and cloned into Agel site in modified pcDNA3.1+myc vector. For the rescue experiments, murine $D b p$ was subcloned into pCS2+ vector between BamHI and SnaBI sites. A $3^{\prime}$ partial zebrafish $d b p$ was PCR amplified. The amplicon was cloned into pCRIITOPO vector (Invitrogen) and was used to generate antisense probe for in situ hybridization. All the primer sequences used are listed in Table 1.

Whole-mount Oil Red-O staining

Embryos at various stages of development were fixed with $4 \%$ paraformaldehyde at $4^{\circ} \mathrm{C}$ overnight, washed 3 times with PBT, and preincubated in $60 \%$ isopropanol for 30 minutes. Embryos were then incubated with freshly filtered $0.3 \%$ solution of Oil Red-O (Sigma) in 60\% isopropanol for 3 hours, rinsed 3 times with PBT and photographed.

In situ hybridization, immunostaining and microscopy In situ hybridization and immunostaining were performed as described previously (Choe et al., 2009). The $d p b$ antisense probe was synthesized using a standard protocol. For immunostaining, antibodies to Znp-1 (1:1,000, DSHB) and acetylated tubulin $(1: 1,000$, Sigma) followed by secondary Alexa Fluorconjugated anti-Mouse antibodies (1:400, Invitrogen) were used. Embryos were imaged with a Leica M165FC microscope equipped with Leica DFC500.

\section{Quantitative RT-PCR}

Total RNAs were prepared from 10 embryos at desired developmental stages using Trizol (Ambion) reagent following manufacturer's instructions. First strand cDNA was synthesized (Roche) and quantitative PCR was performed. All the primer sequences used are listed in Table 1. Gene expression levels relative to that of $\beta$-actin, is presented as an average of values from three independent experiments. Statistical significance was determined in Microsoft Excel, and $p<0.05$ was considered to be significant.

\section{RESULTS}

$d b p$ is maternally deposited during zebrafish development We first compared protein sequence of zebrafish Dbp with that of human or mouse, and found that DBP is well conserved in vertebrates, especially in the conserved, catalytic domains (Fig. 1A). We next examined $d b p$ expression during zebrafish embryogenesis by performing quantitative RT-PCR. As detected at 2-4 cell-stage embryos, Zebrafish $d b p$ mRNA was found to be maternally delivered (Fig. 2A). Expression level of maternal $d b p$ mRNAs decreased at the onset of zygotic transcription. As embryogenesis proceeded, expression of $d b p$ mRNAs showed recovery and began to increase gradually. We also performed in situ hybridization to examine whether $d b p$ expression is restricted to a specific cell type during zebrafish embryogenesis. 
A

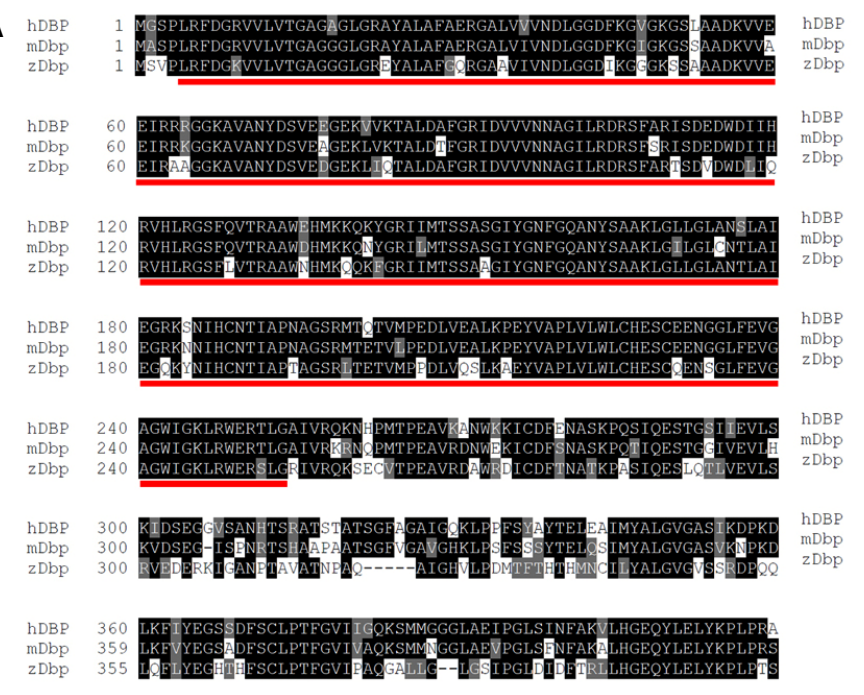

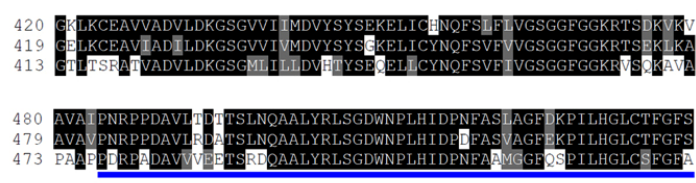
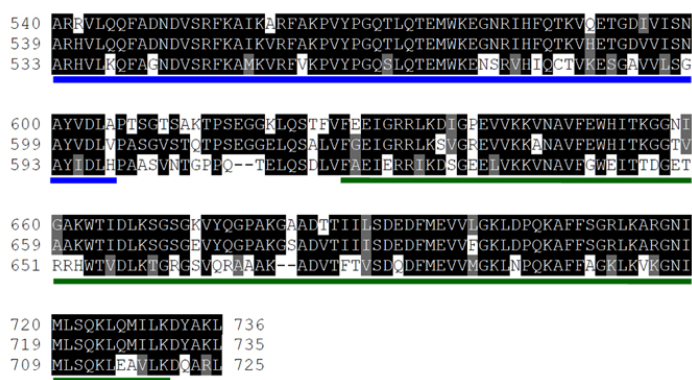

B

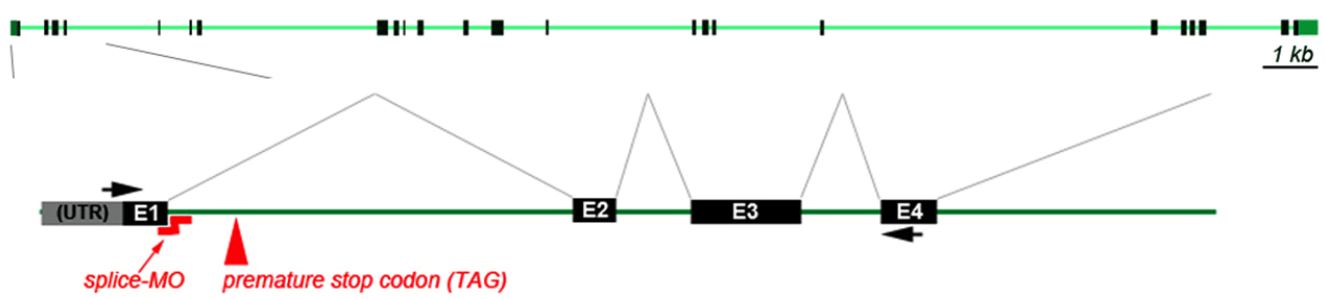

Fig. 1. Amino acid sequence alignment of human, mouse, and zebrafish DBP homologs and genomic structure of zebrafish dbp. (A) Amino acid sequence alignment of human, mouse, and zebrafish homologs of DBP using ClustalW. Amino acids conserved in at least two species are highlighted in black and similar amino acids in gray. Conserved domains are underlined in red (NADB_Rossmann fold), blue (Hotdog fold) and green (SCP2). (B) Diagram depicting genomic structure of zebrafish $d b p$ and the target site of $d b p$ splicing-blocking morpholino. $d b p$ splicing-blocking $\mathrm{MO}$ interferes with the splicing event by blocking the splice donor region of the exon-intron boundary, which may result in translation of aberrant, truncated 27 amino acids from the intron sequences. A primer set (black arrows) was used to test the efficacy of $d b p$ splicingblocking MO.

In situ hybridization with $d b p$ antisense probe showed $d b p$ was ubiquitously expressed at least from 2-cell stage of embryos (not shown), consistent with maternal deposition of $d b p$ mRNA determined by quantitative RT-PCR.

\section{dbp knockdown impairs embryonic development}

We used a morpholino (MO)-based knockdown approach to determine the role of Dbp in zebrafish embryogenesis. A splicing-blocking $\mathrm{MO}$ effectively reduced the amount of mature $d b p$ mRNA without generating any mis-spliced transcripts that probably underwent nonsense-mediated decay, a well-known process in response to a blockage of a splicing event (Figs. 1B and $2 \mathrm{~B}$ ). Both translation-blocking and splicing-blocking MOs generated morphologically abnormal embryos starting from 1 day post-fertilization (dpf) (Figs. 2C and 2D). Embryos occasionally exhibited curved body axis (not shown). At 2 dpf and later during development, edema was clearly visible in the pericardium and around the yolk. As compared to wild type embryos, the size of the yolk was noticeably larger in $d b p$ knockdown embryos (Figs. $2 \mathrm{E}-2 \mathrm{H}$ ). Therefore, defective $\beta$-oxidation in the peroxisome resulting from the $d b p$ knockdown likely impaired normal embryogenesis in zebrafish.

dbp knockdown suppresses yolk consumption

Unlike mammals where embryos in utero receive nutrients from mother through the umbilical cord, zebrafish embryos are limited in their energy source to the yolk components for the initial 5 days of development (Flynn et al., 2009). Zebrafish embryos consume the yolk, eventually depleting it by the time they are ready to be fed at about 6-7 dpf. We used Oil Red-O staining to detect neutral lipids stored in developing zebrafish embryos, and found that consistent with a previous report (Schlegel and Stainier, 2006), neutral lipids were largely located in the yolk and tissues such as brain, heart, and muscles for the first 3 days of normal embryonic development (Fig. 3A). Normally developing embryos consumed most yolk lipids by $4 \mathrm{dpf}$ and almost completely depleted them at $5 \mathrm{dpf}$ (Figs. $3 \mathrm{C}$ and $3 \mathrm{E})$. In contrast, $d b p$ knockdown embryos failed to use much of the yolk lipids, as was evident starting at $3 \mathrm{dpf}$ (Figs. 3B, 3D, and $3 \mathrm{~F})$. Therefore, peroxisomal $\beta$-oxidation may constitute a key component for yolk consumption that supports embryonic growth during development.

dbp knockdown alters neuronal development

DBP deficiency in humans has been shown to result in neuropathy including demyelination (Moller et al., 2001; van Grunsven et al., 1999). We explored whether $d b p$ knockdown generates neuronal abnormality with characteristics similar to that of DBP deficiency in human. Analysis of transgenic zebrafish expressing green fluorescent protein (GFP) in primary motor 
A
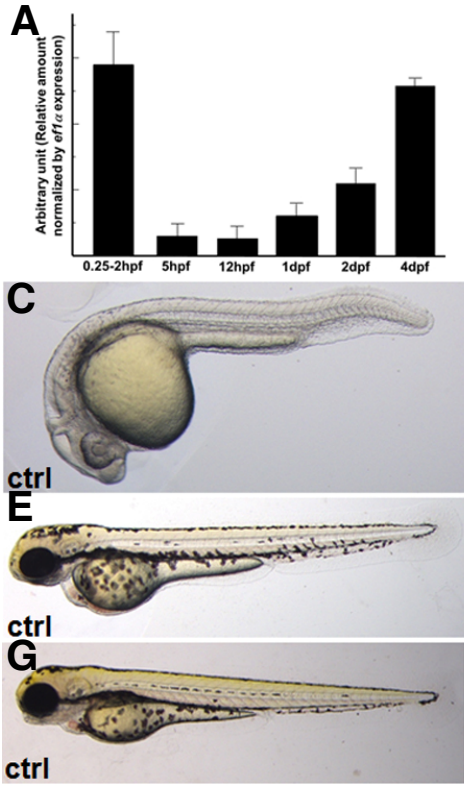

B
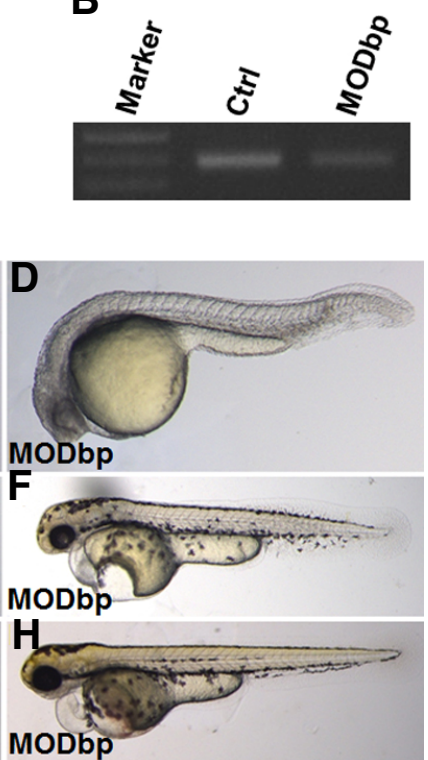

Fig. 2. $d b p$ expression is essential for zebrafish embryogenesis. (A) dbp expression during development. Quantitative RT-PCR was used to assay $d b p$ expression during development. The highest expression of $d b p$ is at 0-2 hpf (maternally deposited) after which it is significantly decreased but increased gradually at $1 \mathrm{dpf}$ onwards. The graph shows relative amount of $d b p$ mRNA normalized by ef1 $\alpha$ expression. (B) $d b p$ splicing-blocking morpholino (MO) efficiently reduced synthesis of mature mRNA. Total RNA extracted from control and MO-injected embryos at 1 dpf were used for CDNA synthesis followed by RT-PCR. A $\sim 390 \mathrm{bp}$ band in the control lane was significantly reduced in the morpholino-injected lane (MODbp), indicating high efficiency of MO. (C-H) dbp knockdown generates morphologically distinct phenotypes. MO-injected embryos displayed phenotypes, including small head, pericardial ede$\mathrm{ma}$, and voluminous yolk compared to control embryos [compare (C, E, and $\mathrm{G}$ ) with (D, F, and $\mathrm{H})$ ]. As embryogenesis continued, phenotypes became more severe $[(C)$ and (D) (1dpf); (E) and (F) (2 dpf); (G) and (H) (3 dpf)]. Embryos are shown in lateral view with anterior to the left.
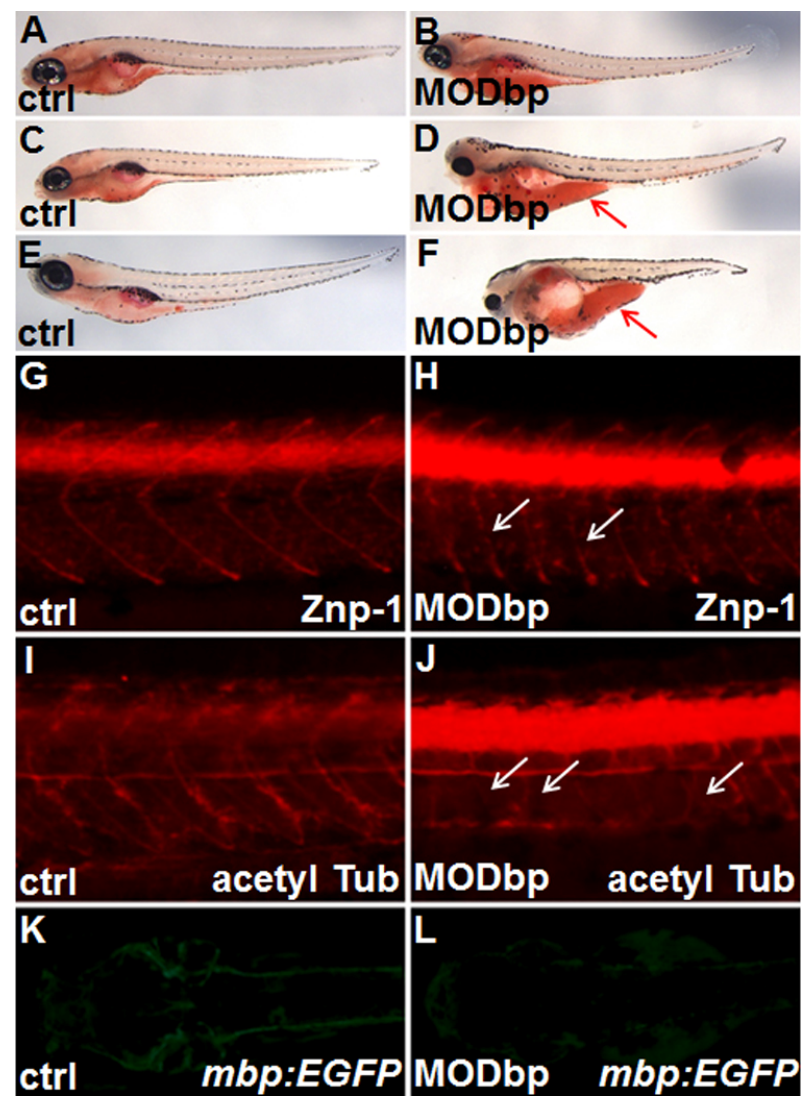

Fig. 3. $d b p$ knockdown affects yolk consumption and neuronal development. (A-F) Embryos with dbp knockdown showed accumulated neutral lipids in the yolk. Oil Red-O staining revealed a significant delay or defect in yolk lipid consumption in dbp knockdown embryos on or after $3 \mathrm{dpf},(\mathrm{A})$ and (B) (3 dpf); (C) and (D) (4 dpf); $(E)$ and $(F)(5 \mathrm{dpf})$. As compared to control embryo, greater quantities of yolk lipids (visualized in red) were retained in a $d b p$ knockdown embryo. Red arrows in (D) and (F) indicate accumulated lipids in the yolk. (G-L) dbp knockdown impaired neuronal development. Immunostaining using anti-Znp-1 antibody (G, H) or antiacetylated tubulin $(\mathrm{I}, \mathrm{J})$ revealed defective neuronal development, such as discontinued axonal projections [indicated by white arrows in $(H)$ ] or suppression of differentiating motor axons [indicated by white arrows in (I)] upon $d b p$ knockdown. (K, L) GFP expression driven by the myelin basic protein $(\mathrm{mbp})$ promoter (indicative of myelinating cells) in an mbp:EGFP transgenic line was significantly reduced following $d b p$ knockdown. Embryos are shown in lateral view with anterior to the left, except in $(K)$ and $(L)$ where embryos are in dorsal view. Partial trunk regions of embryos are shown in (GJ). neurons (islet1:GFP) of the central nervous system (CNS) indicated normal GFP expression in $d b p$ knockdown embryos (not shown). This suggested that Dbp might not be required for motor neuron specification in the CNS. However, Znp1, an axonal marker, was affected, featuring discontinued CaP pro- jections occasionally in the trunk (Figs. $3 \mathrm{G}$ and $3 \mathrm{H}$ ). Additionally, severe suppression of motor axon differentiation in the trunk was also detected in dbp knockdown embryos (Figs. 3l and 3J). Further, GFP expression driven by the promoter of myelin basic protein in Schwann cells of the control mbp:EGFP transgenic 


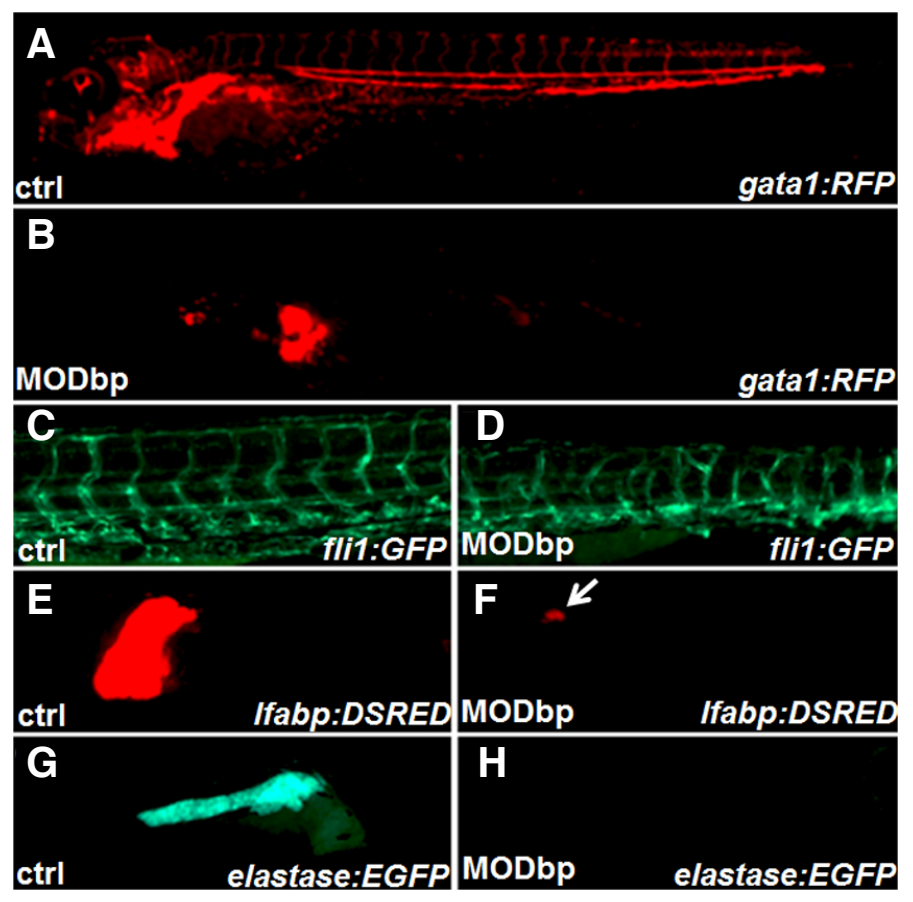

Fig. 4. $d b p$ knockdown affects development of blood, blood vessels and digestive organs. Embryos carrying different transgenes were used to determine tissue-specific effects upon $d b p$ knockdown. (A-D) dbp knockdown affects red blood cell development and blood vessel patterning. Upon $d b p$ knockdown, blood cells were severely reduced [shown by gata1:RFP transgenic line, (A) and (B)] and blood vessels were formed abnormally [shown by fli1:EGFP, (C) and (D)]. (E-H) dbp knockdown on doubly transgenic fish line (Ifabp: DSRED in liver and elastase:GFP in pancreas) shows significantly reduced liver size $(F)$ and absence of pancreas $(\mathrm{H})$. Representative embryos at $3 \mathrm{dpf}$ are shown in lateral view with anterior to the left, except $(G)$ and $(H)$ where presented in lateral view with anterior to the right.

A

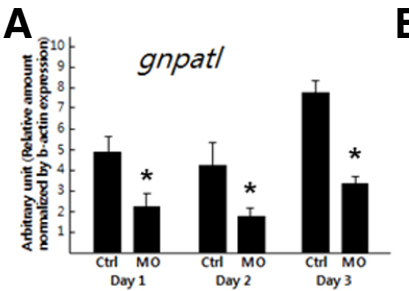

D

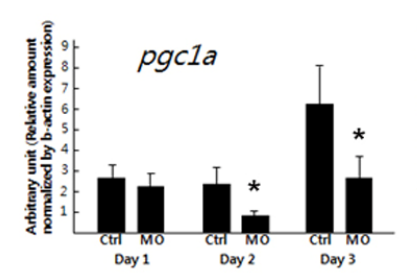

E
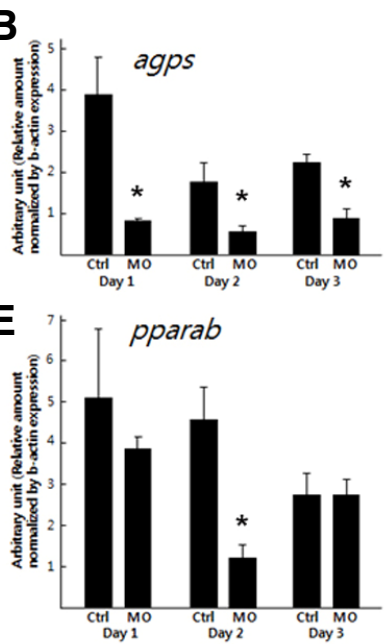

G

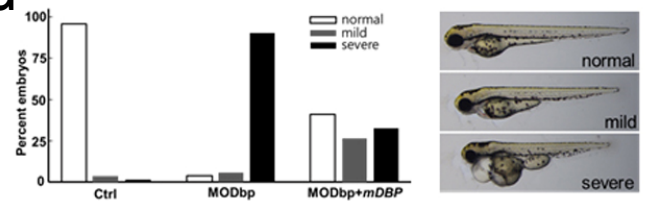

$\mathbf{F}$

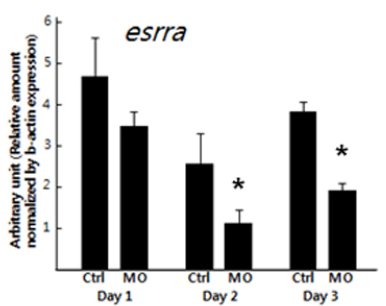

C

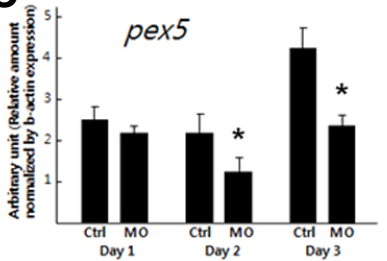

in the right were counted from three independent experiments and presented as percentage of total injected embryos. samples. (G) DBP is functionally conserved between mice and zebrafish. Rescue experiment was performed by co-injecting murine Dbp mRNA together with dbp MO. Embryos belonging to each category of phenotypic severity shown

Fig. 5. Knockdown of dbp affects expression of genes involved in chondrial biogenesis. (A-F) Quantitative RT-PCR was used to assay expression of genes involved in ether phospholipid synthesis: gnpat 1 (A) and agps (B), a gene in peroxand genes in mitochondrial biogenesis: pgc1a (D), pparab (E) and esrra (F). dbp knockdown altered the expression of genes in involved in ether phospholipid synthesis starting at $1 \mathrm{dpf}(\mathrm{A}-\mathrm{B})$ and that of genes in peroxisomal protein transport or mitochondrial biogenesis at $2 \mathrm{dpf}$. * indicates statistical significance $(p<$
$0.05)$ between control and $d b p \mathrm{MO}$ lembryos. zebrafish was almost abrogated in dbp knockdown embryos (Figs. 3K and $3 \mathrm{~L}$ ). These results strongly suggest that the zebrafish Dbp may act similarly to that in mice and humans to promote neuronal development and integrity (Moller et al., 2001; van Grunsven et al., 1999; Verheijden et al., 2013). dbp knockdown impairs organogenesis and tissue development

While using various transgenic lines to detect tissue-specific abnormalities upon $d b p$ knockdown, we discovered several developmental programs being impaired in addition to observ- 
ing the phenotypes expected based on that found in mice and human (Baes and Van Veldhoven, 2012; Huyghe et al., 2006; Van Veldhoven et al., 2010). For examples, the numbers of red blood cells were reduced (detected by the gata1:RFP line, Figs. $4 \mathrm{~A}$ and $4 \mathrm{~B}$ ) and blood vessels were abnormally patterned (by the fli1:EGFP line, Figs. 4C and 4D) in dbp knockdown embryos. Additionally, abnormal cartilage formation was also observed (not shown). Starting from $3 \mathrm{dpf}$, both liver and pancreas (by the Ifabp:DSRED and elastase:GFP lines, respectively) developed poorly in dbp knockdown embryos (Figs. 4E-4H). In situ hybridization showed that expression of foxa3, a marker of early endodermal organs, was severely reduced or absent in liver and pancreas of $d b p$ knockdown embryos (not shown). These results demonstrated that $d b p$ knockdown in zebrafish impaired a number of developmental programs including those that have not been previously reported in connection with peroxisome dysfunction in humans or mice.

\section{dbp knockdown alters expression of various genes involved in peroxisomal pathways and mitochondrial biogenesis}

Since $d b p$ knockdown may also affect other peroxisomal functions that confer abnormality on developing embryos, we next examined expression of various genes important in major peroxisomal pathways. We found no differences in the expression of other enzymes in the $\beta$-oxidation pathway including acox1, $s c p 2 a$, and $s c p 2 b$ between wild type and DBP knockdown embryos (not shown). In contrast, mRNA expression of the enzymes involved in the synthesis of ether phospholipids, such as gnpatl and agps, was significantly down-regulated in $d b p$ knockdown embryos at $1 \mathrm{dpf}$ (Figs. 5A and 5B). We also found that expression of pex5, the major carrier of peroxisometargeted proteins, was significantly down-regulated starting from 2 dpf during development (Fig. 5C). Similarly, dbp knockdown also down-regulated expression of transcription factors known as upstream regulators of mitochondrial biogenesis (Figs. $5 \mathrm{D}-5 \mathrm{~F})$. These results suggested the presence of cross-talk between different functions of peroxisome. Additionally, our results suggest that dysfunctional peroxisome may affect other metabolic organelles such as mitochondria, potentially inducing phenotypic exacerbation as observed at later stages of development.

\section{Exogenous murine Dbp can rescue embryonic} phenotypes resulting from $d b p$ knockdown in zebrafish During the course of experiments, we noticed that morphological phenotypes were closely related to other abnormalities described. Since we found a significant homology between mice and zebrafish DBP protein sequences (Fig. 1), we used murine $D b p$ to rescue morphological defects observed in $d b p$ knockdown embryos. As expected, murine Dbp over-expression efficiently rescued developmental abnormalities caused by $d b p$ knockdown in zebrafish embryos (Fig. 5G). These results demonstrated that the observed phenotypes were specifically induced by $d b p$ knockdown. Our results clearly indicated conserved functions of DBP in zebrafish and mammals.

\section{DISCUSSION}

In the study reported here, we assessed whether zebrafish can be used as an in vivo model for examining peroxisome functions by examining developmental roles of $\mathrm{Dbp}$. We found that $d b p$ knockdown in zebrafish gave rise to a phenotypic spectrum similar to that found in human patients. In particular, clinical symptoms such as growth retardation, neuropathy, and craniofacial disfiguration in human patients were recapitulated in dbp-knockdown zebrafish embryos that displayed reduced yolk consumption, abnormal neuronal development, and cartilage malformation. Interestingly, phenotypes not found in previous mammalian models were also observed. In addition to defective blood and vessel formation, we found that $d b p$ knockdown nearly blocked digestive system development. Although cases of hepatomegaly associated with peroxisome dysfunction in humans have been reported (Roels et al., 1993), severe defects in the development of digestive organs have not been observed in other organisms. Our results illustrate the usefulness of the zebrafish system in analyzing the role of metabolic pathways in development and diseases.

While DBP deficiency in mammals leads to compensatory activation of several peroxisomal enzymes, dbp knockdown in zebrafish did not affect expression of enzymes in the peroxisomal $\beta$-oxidation pathway. Instead, we found decreased expression of genes involved in other peroxisomal pathways, such as the import of peroxisomal matrix proteins and synthesis of ether phospholipids. This phenotypic difference between zebrafish and mammals may at least in part be due to the differences in stages of development analyzed. We found $d b p$ knockdown-mediated transcriptional changes as early as $1 \mathrm{dpf}$ when most organs were yet to be formed, while DBP-deficient mice or human patients were examined at single organ/tissue (liver, brain, plasma in most cases) levels at several weeks of postnatal age (Baes et al., 2000; Ferdinandusse et al., 2005; Huyghe et al., 2006; Moller et al., 2001; Suzuki et al., 1997; van Grunsven et al., 1998; 1999). Accordingly, we found that phenotypes of developing zebrafish embryos with $d b p$ knockdown described in this study were far more severe than those reported in $D B P$-deficient mammals.

We also analyzed the possibility that impaired $\beta$-oxidation in the peroxisome due to $d b p$ knockdown may interfere with mitochondrial biogenesis, analogous to previous reports of abnormal mitochondrial morphologies observed in instances of peroxisome dysfunctions (Baes et al., 1997; Baumgart et al., 2001; Dirkx et al., 2005; Muller-Hocker et al., 1984), dbp knockdown decreased expression of pgc1 $\alpha$, ppar $\alpha$ and esrra starting at 2 dpf. Given the importance of these genes regulating a number of pathways in mitochondria and peroxisomes, this result suggests that metabolic disturbance induced by peroxisomal $\beta$ oxidation may trigger to transcriptional suppression of upstream factors by an unknown feedback mechanism. Nonetheless, our results suggest that a functional link between the peroxisome and other metabolic organelles including mitochondria may be a critical factor for normal animal development.

In conclusion, the zebrafish system with metabolism confined within itself during early development may be an attractive animal model to analyze the molecular basis and physiological consequences of peroxisomal dysfunctions.

\section{ACKNOWLEDGMENTS}

We thank Drs. C-H Kim, H-C Park, Y-K Bae, Y Kee and S-Y Choi, and the Zebrafish Organogenesis and Mutant Bank for providing transgenic lines and DNA constructs. We also thank Developmental Studies Hybridoma Bank for providing antibody (lowa, USA). This work was supported by the National Research Foundation of Korea (NRF) grants funded by the Korean government [Ministry of Science, ICT \& Future Planning (MSIP)]: (No. 2011-0028866) and (No. 2011-0030719). The authors declare no conflict of interests. 


\section{REFERENCES}

Baes, M., and Van Veldhoven, P.P. (2012). Mouse models for peroxisome biogenesis defects and b-oxidation enzyme deficiencies. Biochim. Biophys. Acta 1822, 1489-1500.

Baes, M., Gressens, P., Baumgart, E., Carmeliet, P., Casteels, M., Fransen, M., Evrard, P., Fahimi, D., Declercq, P.E., Collen, D. et al. (1997). A mouse model for Zellweger syndrome. Nat. Genet. 17, 49-57.

Baes, M., Huyghe, S., Carmeliet, P., Declercq, P.E., Collen, D., Mannaerts, G.P., and Van Veldhoven, P.P. (2000). Inactivation of the peroxisomal multifunctional protein-2 in mice impedes the degradation of not only 2-methyl branched fatty acids and bile acid intermediates but also of very long chain fatty acids. J. Biol. Chem. 275, 16329-16336.

Baumgart, E., Vanhorebeek, I., Grabenbauer, M., Borgers, M., Declercq, P.E., Fahimi, H.D., and Baes, M. (2001). Mitochondrial alterations caused by defective peroxisomal biogenesis in a mouse model for Zellweger syndrome (PEX5 knockout mouse). Am. J. Pathol. 159, 1477-1494.

Choe, S.K., Lu, P., Nakamura, M., Lee, J., and Sagerstrom, C.G. (2009). Meis cofactors control HDAC and CBP accessibility at Hox-regulated promoters during zebrafish embryogenesis. Dev. Cell. 17, 561-567.

Choe, S.K., Zhang, X., Hirsh, N., Straubhaar, J., and Sagerstrom, C.G. (2011). A screen for hoxb1-regulated genes identifies ppp1r14al as a regulator of rhombomere 4 Fgf-signaling center. Dev. Biol. 358, 356-367.

Dirkx, R., Vanhorebeek, I., Martens, K., Schad, A., Grabenbauer, M., Fahimi, D., Declercq, P., Van Veldhoven, P.P., and Baes, M. (2005). Absence of peroxisomes in mouse hepatocytes causes mitochondrial and ER abnormalities. Hepatology 41, 868-878.

Faust, P.L., Su, H.M., Moser, A., and Moser, H.W. (2001). The peroxisome deficient PEX2 Zellweger mouse: pathologic and biochemical correlates of lipid dysfunction. J. Mol. Neurosci. 16, 289-297.

Ferdinandusse, S., Denis, S., Overmars, H., Van Eeckhoudt, L., Van Veldhoven, P.P., Duran, M., Wanders, R.J., and Baes, M. (2005). Developmental changes of bile acid composition and conjugation in L- and D- bifunctional protein single and double knockout mice. J. Biol. Chem. 280, 18658-18666.

Flynn, E.J. $3^{\text {rd }}$, Trent, C.M., and Rawls, J.F. (2009). Ontogeny and nutritional control of adipogenesis in zebrafish (Danio rerio). J. Lipid Res., 50, 1641-1652.

Huyghe, S., Mannaerts, G.P., Baes, M., and Van Veldhoven, P.P. (2006). Peroxisomal multifunctional protein-2: the enzyme, the patients and the knockout mouse model. Biochim. Biophys. Acta 1761, 973-994.

Islinger, J., Grille, S., Fahimi, H.D., and Schrader, M. (2012). The peroxisome: an update on mysteries. Histochem. Cell. Biol. 137, 547-574.

Kimmel, CB., Ballard, W.W., Kimmel, SR., Ullmann, B., and Schilling, T.F. (1995). Stages of embryonic development of the zebrafish. Dev. Dyn. 203, 253-310.

Lieschke, G.J., and Currie, P. (2007). Animal models of human disease: zebrafish swim into view. Nat. Rev. Genet. 8, 353-367.

Mehtälä, M.L., Lensink, M.F., Pietikäinen, L.P., Hiltunen, J.K., and Glumoff, T. (2013). On the molecular basis of D-bifunctional protein deficiency type III. PLoS One 8, e53688.

Moller, G., Van Grunsven, E.G., Wanders, R.J., and Adamski, J. (2001). Molecular basis of D-bifunctional protein deficiency. Mol. Cell. Endocrinol. 171, 61-70.

Muller-Hocker, J., Walther, J.U., Bise, K., Pongratz, D., and Hubner, G. (1984). Mitochondrial myopathy with loosely coupled oxidative phosphorylation in a case of Zellweger syndrome. A cytochemical-ultrastructural study. Virchows. Arc. B Cell. Pathol. Incl. Mol. Pathol. 45, 125-138.

Roels, F., Espeel, M., Poggi, F., Mandel, H., Van Maldergem, L., and Saudubray, J.M. (1993). Human liver pathology in peroxisomal diseases: a review including novel data. Biochimie 75 , 281-292.

Schlegel, A., and Stainier, D.Y. (2006). Microsomal triglyceride transfer protein is required for yolk lipid utilization and absorption of dietary lipids in zebrafish larvae. Biochemistry 45, 15179-15187.

Suzuki, Y., Jiang, L.L., Souri, M., Miyazawa, S., Fukuda, S., Zhang, Z., Une, M., Shimozawa, N., Kondo, N., Orii, T., et al. (1997). D3-Hydroxyacyl-CoA dehydratase/D-3-hydroxyacyl-CoA dehydrogenase bifunctional protein deficiency: a newly identified peroxisomal disorder. Am. J. Hum. Genet. 61, 1153-1162.

Thoms, S., Gronborg, S., and Gartner, J. (2009). Organelle interplay in peroxisomal disorders. Trends Mol. Med. 15, 293-302.

Van Grunsven, E.G., Van Berkel, E., and ljlst, L. (1998). Peroxisomal D-hydroxyacyl-CoA dehydrogenase deficiency: resolution of the enzyme defect and its molecular basis in bifunctional protein deficiency. Proc. Natl. Acad. Sci. USA 95, 2128-2133.

Van Grunsven, E.G., Mooijer, P.A., Aubourg, P., and Wanders, R.J. (1999). Enoyl-CoA hydratase deficiency: identification of a new type of D-bifunctional protein deficiency. Hum. Mol. Genet. 8, 1509-1516.

Van Veldhoven, P.P., (2010). Biochemistry and genetics of inherited disorders of peroxisomal fatty acid metabolism. J. Lipid Res. 51, 2863-2895.

Verheiiden, S., Bottelbergs, A., Krysko, O., Krysko, D.V., Beckers, L., De Munter, S., Van Veldhoven, P.P., Wyns, S., Kulik, W. Nave, K.A., et al. (2013). Peroxisomal multifunctional protein-2 deficiency causes neuroinflammation and degeneration of purkinje cells independent of very long chain fatty acid accumulation. Neurobiol. Dis. 58, 258-269. 